\title{
Isenthalpic oscillations with quadratic damping in saturated two-phase fluids
}

\author{
J. V. Madison \\ Ebara International Corporation, Sparks, Nevada, USA
}

\begin{abstract}
Saturated two-phase fluid flows are often subject to pressure induced oscillations. Due to compressibility the vapour bubbles act as a spring with an asymmetric non-linear characteristic. The volume of the vapour bubbles increases or decreases differently if the pressure fluctuations are compressing or expanding; consequently, compressing pressure fluctuations in a two-phase pipe flow cause less displacement in the direction of the pipe flow than expanding pressure fluctuations. The displacement depends on the ratio of liquid to vapour, the ratio of pressure fluctuations over average pressure, on the exciting frequency of the pressure fluctuations and the damping factor.

In addition, pressure fluctuations in saturated vapour bubbles cause condensation and evaporation within the bubbles and change periodically the liquid to vapour ratio and influence the dynamic parameters for the oscillation. The oscillations conform to an isenthalpic process at constant enthalpy with no heat transfer and no exchange of work. Due to friction forces the oscillations are subjected to quadratic fluid damping.

The paper describes the governing non-linear equation for quadratically damped oscillations in saturated two-phase fluids with condensation and evaporation, and presents steady state approximate solutions for free and for pressure induced oscillations. Resonance criteria and stability are discussed.

Keywords: two-phase flow, isenthalpic oscillations, quadratic fluid damping, saturated fluid, condensation, evaporation, steady state solutions.
\end{abstract}

\section{Introduction}

A saturated two-phase fluid is a mixture of liquid and vapour. The volumetric ratio $\mathrm{R}$ between the vapour and the liquid portion is defined as 


$$
R=\frac{V_{v}}{V_{v}+V_{L}}
$$

where $R=0$ for pure liquid and $R=1.0$ for pure vapour. In standard books for Thermodynamics [1], the ratio $R$ is called the quality of the fluid with the symbol $\mathrm{x}$ instead of $R . V_{V}$ is the vapour volume and $V_{L}$ is the liquid volume. With very good accuracy, the liquid portion can be assumed to be incompressible when compared to the compressible vapour portion. The vapour portion follows approximately the ideal gas equation of state with the vapour volume $V_{V}$ inversely proportional to the pressure $p$ at constant temperatures. Pressure changes $\Delta p$ and volumetric changes are related by (2)

$$
\frac{p+\Delta p}{p}=\frac{V_{V}}{V_{V}+\Delta V_{V}}
$$

Eqn (2) may also be written in the form:

$$
\Delta p=-p \frac{\Delta V_{V}}{V_{V}+\Delta V_{V}}
$$

Within a controlled volume of a two-phase fluid defined as the sum of $V_{V}$ and $V_{L}$ equals one. The volumetric vapour content is equal to

$$
V_{v}=R
$$

The amount of condensing and evaporating fluid depends on the pressure change, and with $r$ as the rate of change the momentary vapour portion $V_{V}$ as a function of the pressure is

$$
V_{v}=R+r \cdot \Delta p .
$$

Fig. 1 shows the asymmetric oscillations in a two-phase pipe flow. For a given absolute pressure difference $\Delta p$ the absolute displacement $\Delta X(t)$ is smaller for compression than for expansion.

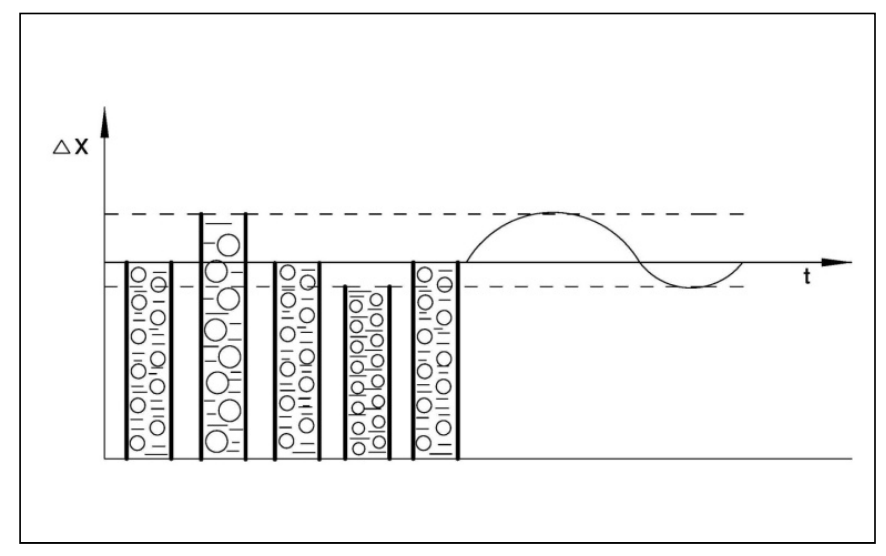

Figure 1: Asymmetric displacement of vapour bubbles for expansion and compression. 
Fig. 2 demonstrates the condensation and evaporation within the vapour bubble for isenthalpic compression and expansion for saturated two-phase fluids, if the slope of the curves $(\mathrm{R}=$ constant) is positive [1]. The slope is positive towards the liquid side of the saturation dome. A pressure increase causes a partial condensation, and a pressure decrease causes a partial evaporation of the vapour bubble.

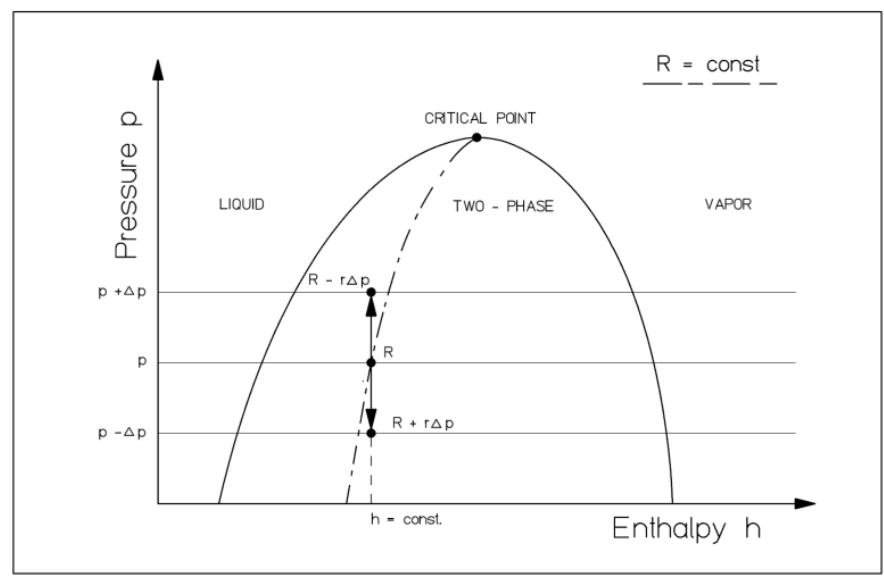

Figure 2: Isenthalpic oscillations with condensation for increasing pressure.

Fig. 3 demonstrates the condensation and evaporation for a negative slope of the $R=$ constant curves. The slope is negative towards the vapour side of the saturation dome, and a pressure increase causes a partial evaporation, and a pressure decrease causes a partial condensation of the vapour bubble.

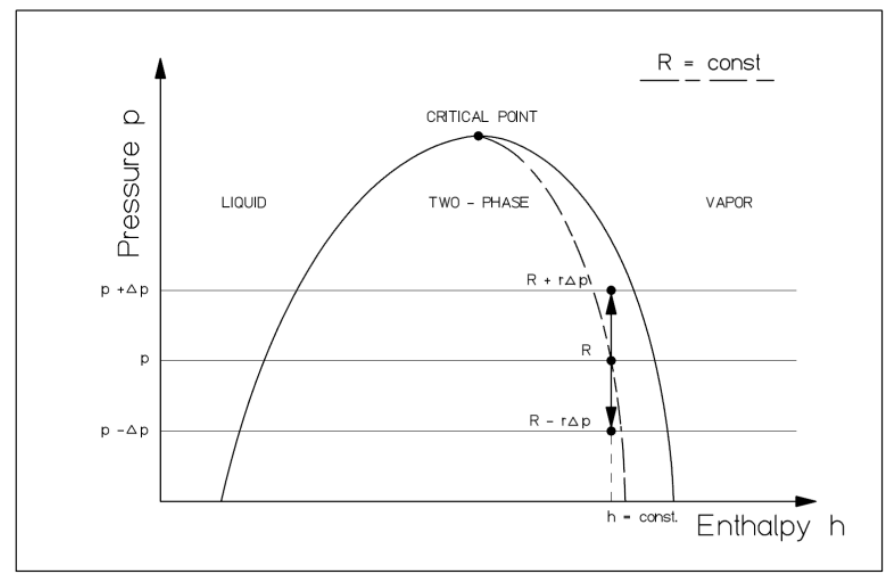

Figure 3: Isenthalpic oscillations with evaporation for increasing pressure. 
Any evaporation makes the spring characteristic of the oscillating system softer and the mass smaller, and any condensation makes the spring characteristic stiffer and the mass larger. These changes of the system characteristics during a periodic oscillation result in complicated non-linear periodic oscillations.

\section{Free isenthalpic oscillations in saturated fluids}

Pressure changes $\Delta p$ act as a force in the axial direction $X$ of the pipe. The compressible vapour volume and the mass of the liquid volume form an oscillating system. The vapour volume acts as an asymmetric spring and generates axial forces non-linear to the displacement $X$ as a function of time $t$.

$$
\Delta p \approx-X \approx-\Delta V_{V}
$$

The density of the vapour is negligibly small compared to the density $\rho$ of the liquid, and the oscillating mass $\mathrm{m}$ within the controlled volume $V_{V}+V_{L}=1$ depends only on the volume $V_{L}$ of the liquid.

$$
m=\rho V_{L}=\rho\left(1-V_{V}\right)=\rho(1-R+r \Delta p)=\rho(1-R-r X)
$$

Eqn (8) is a non-linear differential equation that models the free oscillation in saturated fluids with condensation and evaporation:

$$
\rho(1-R-r X(t)) \frac{d^{2} X(t)}{d t^{2}}+p \frac{X(t)}{R+r X(t)+X(t)}=0 .
$$

For very small displacements, $X(t)$, the oscillating system can be considered as a linear harmonic oscillator,

with the natural frequency,

$$
\rho(1-R) \frac{d^{2} X(t)}{d t^{2}}+p \frac{X(t)}{R}=0 .
$$

$$
f_{N}=\sqrt{\frac{p}{\rho R(1-R)}}
$$

and

$$
\frac{d^{2} X(t)}{d t^{2}}+f_{N}^{2} X(t)=0
$$

The non-linear differential equation can be written now in term of the natural frequency and transforms into:

$$
(1-R-r X(t)) \frac{d^{2} X(t)}{d t^{2}}+f_{N}^{2} \frac{X(t)}{R+(1+r) X(t)}=0 .
$$

Steady state approximate solutions for free isenthalpic oscillations are included in the solutions for the pressure induced oscillations. 


\section{Forced pressure induced oscillations in saturated fluids with quadratic damping}

In the case of forced oscillations with harmonic excitation the non-linear differential equations transforms into:

$$
(1-R-r X(t)) \frac{d^{2} X(t)}{d t^{2}}+f_{N}^{2} \frac{R(1-R) X(t)}{R+(1+r) X(t)},=G f^{2} \cos (f t+\varphi)
$$

The exciting force $G f^{2}$ depends on the exciting amplitude $G$ and the square of the exciting frequency $f$. Friction forces generated by fluids are opposed to the velocity vector and proportional to the square of the displacement velocity multiplied by the damping factor $\mathrm{D}$. In damped oscillations there is a phase angle $\varphi$ between the harmonic excitation and the periodic oscillation.

The quadratic fluid damping force $Q_{D}$ caused by the fluid friction is described with

$$
Q_{D}=-D \frac{d X(t)}{d t}\left|\frac{d X(t)}{d t}\right|
$$

In the case of a forced oscillation with harmonic excitation and quadratic fluid damping the following non-linear differential equation describes the pressureinduced isenthalpic oscillations in saturated two-phase fluids

$$
(1-R-r X(t)) \frac{d^{2} X(t)}{d t^{2}}+f_{N}^{2} \frac{R(1-R) X(t)}{R+(1+r) X(t)},=G f^{2} \cos (f t+\varphi)-D \frac{d X(t)}{d t}\left|\frac{d X(t)}{d t}\right| .
$$

This highly non-linear differential equation cannot be solved by analytical methods and numerical or approximation methods have to be applied. The method described by A. Kimmel [3] using Fourier coefficients for the periodic error is applied to generate an approximate analytical solution for the steady state case.

If $X(t)$ is an approximate solution, then $E(t)$ is the function of the error for this approximate solution $X(t)$ :

$$
E(t)=(1-R-r X(t)) \frac{d^{2} X(t)}{d t^{2}}+D \frac{d X(t)}{d t}\left|\frac{d X(t)}{d t}\right|,+f_{N}^{2} \frac{R(1-R) X(t)}{R+(1+r) X(t)}-f^{2} \cos (f t+\varphi) .
$$

The periodic functions of the error $E(t)$ can be expanded in a Fourier series and the Fourier coefficients $a_{k}$ and $b_{k}$ of the periodic error are determined by (17) and (18).

$$
\begin{aligned}
& a_{k}=\int_{0}^{2 \pi} E(t) \cos (k f t) d(f t) . \\
& b_{k}=\int_{0}^{2 \pi} E(t) \sin (k f t) d(f t) .
\end{aligned}
$$


By setting certain $a_{k}$ and $b_{k}$ equal to zero, the error for the $k$-th term of the Fourier series $E(t)$ becomes zero. The approximate solution $X(t)$ is then correct for the $k$-th term of the function $E(t)$.

\section{Approximate solution}

For the given differential equation with forced oscillation through harmonic excitation $G f^{2} \cos (f t+\varphi)$ the approximate solution $X(t)$ corresponds to the same exciting frequency $f$ but with a different amplitude $\mathrm{A}$ and with a phase angle $\varphi$ between excitation and approximate solution.

$$
X(t)=S+A \cos (f t) .
$$

This is part of the Fourier series with $1 / 2 a_{0}=S$ and $a_{1}=A$ and all other Fourier coefficients are equal to zero. By substituting $X(t)$ into the differential equation the Fourier coefficients $a_{0}$ and $a_{1}$ of the error, $E(t)$ can be determined by integration:

$$
\begin{aligned}
& -f^{2}(\mathrm{G} \cos (f t+\varphi)+A \cos (f t)(1-R-r(S+A \cos (f t)))) \\
& +D f^{2} A \sin (f t)|A \sin (f t)|+f_{N}^{2} \frac{R(1-R)(S+A \cos (f t))}{R+(1+r)(S+A \cos (f t))}
\end{aligned}
$$

Integration of (20) leads to the functions (21) and (22) for $a_{0}$ and $a_{1}$ respectively, and for $\mathrm{b}_{1}$ to the function (23).

$$
\begin{aligned}
& a_{0}=a_{0}\left(A, S, f, \varphi, f_{N}, R, r, D, G\right)=0 . \\
& a_{1}=a_{1}\left(A, S, f, \varphi, f_{N}, R, r, D, G\right)=0 . \\
& b_{1}=b_{1}\left(A, S, f, \varphi, f_{N}, R, r, D, G\right)=0 .
\end{aligned}
$$

Setting all three functions (21), (22) and (23) equal to zero produces the approximate solutions for $\mathrm{A}, \mathrm{S}$ and $\varphi$ as a function of the exciting frequency $f$, the exciting force $G$ and the damping factor $\mathrm{D}$ determined by the vapour to liquid ratio $R$ and the condensation and evaporation parameter $r$. The coefficient $S$ can be determined from $a_{0}=0$ with (21).

$$
S=-\frac{R}{1+r}+\sqrt{A^{2}+\frac{4(1-R)^{2} \mathrm{R}^{4}}{(1+r)^{2}\left(A^{2} f_{S}^{2} r(1+r)+2(1-R) R\right)^{2}}} .
$$

The scaled frequency $f_{S}$ is the ratio of exciting frequency $f$ over natural frequency $f_{N}$.

$$
f_{S}^{2}=f^{2} / f_{N}^{2}
$$

Eliminating $\varphi$ using functions $a_{1}$ and $b_{1}$ generates the implicit function (26) for $A, S$ and $f_{S}$.

$$
\left(A(-1+R+r S)+\frac{2(1-R) R^{2}\left(R+S+r S-\sqrt{(R+S+r S)^{2}-A^{2}(1+r)^{2}}\right)}{A f_{S}^{2}(1+r)^{2} \sqrt{(R+S+r S)^{2}-A^{2}(1+r)^{2}}}\right)^{2}+\left(\frac{8 A^{2} D}{3 \pi}\right)^{2}=G^{2} .
$$


Substituting eqn (24) into eqn (26) the solution for $\mathrm{A}$ and $f_{S}$ is found as an implicit function.

Solutions of the functions $a_{0}, a_{1}$ and $b_{1}$ are the stable steady state solutions for isenthalpic oscillations with condensation and evaporation in saturated twophase fluids with quadratic damping.

The phase angle $\varphi$ between the harmonic excitation and the periodic oscillation depends on $A, D$ and $G$.

$$
\varphi=\arcsin \left(\frac{8 D A|A|}{3 \pi G}\right)
$$

The range of the phase angle is $0 \leq \varphi \leq \pi$ for

$$
-3 \pi G \leq 8 D A|A| \leq 3 \pi G \text {. }
$$

\section{Approximate solution examples}

Steady state oscillations with quadratic damping in saturated two-phase fluid exist only with forced excitation.

A typical forced oscillation spectrum with $\mathrm{G}=0.5$ and $\mathrm{R}=0.5$ with a positive quality change rate of $r=0.1$ and damping factors of $\mathrm{D}=0, \mathrm{D}=0.25, \mathrm{D}=0.5$ and $\mathrm{D}=2.0$ are shown in Fig. 4 .

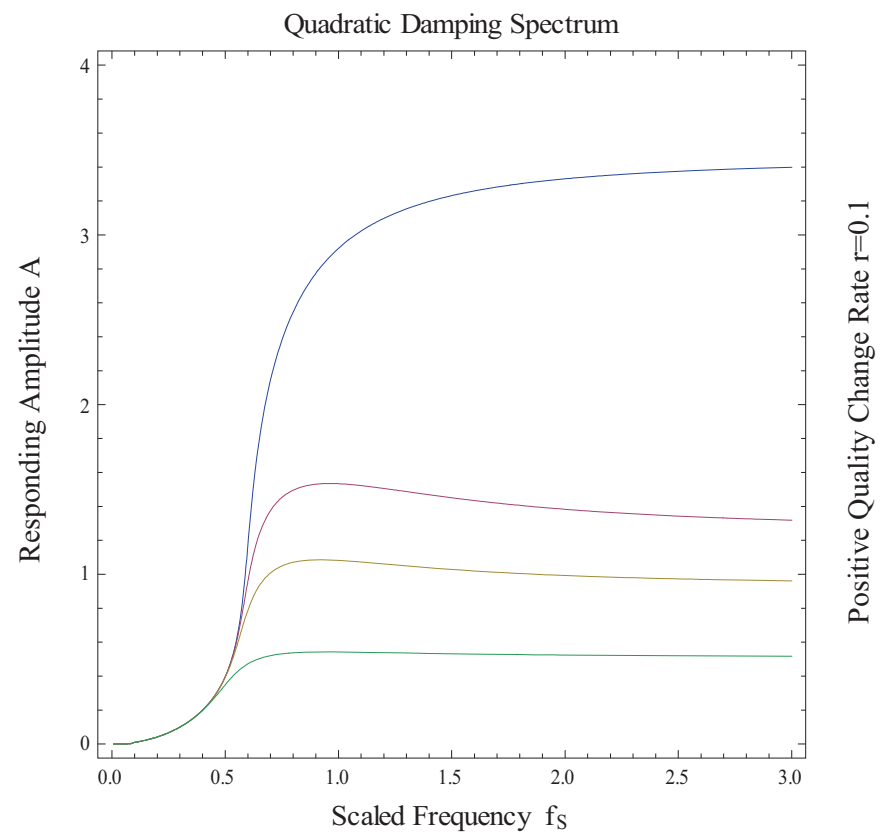

Figure 4: Forced oscillations with quadratic damping and positive quality change rate.

Fig. 5 shows the oscillation spectrum for the same parameters $G, R$, and D as in Fig. 4, but for a negative quality change rate of $r=-0.1$. 
Fig. 6 demonstrates the oscillation spectrum for the same parameters $G, R$, and D as in Fig. 4 and 5, but for a constant quality value with the quality change rate of $r=0$.

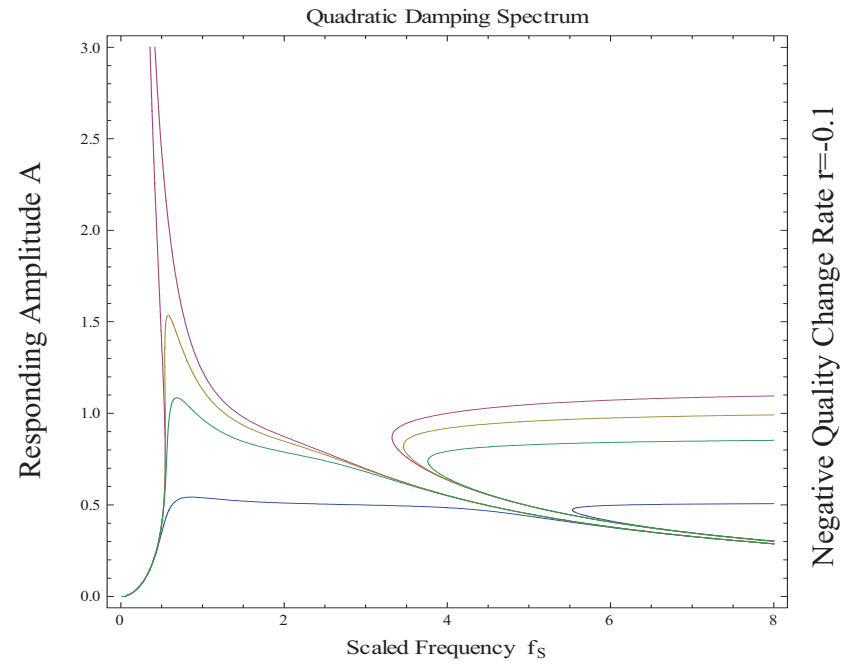

Figure 5: Forced oscillations with quadratic damping and negative quality change rate.

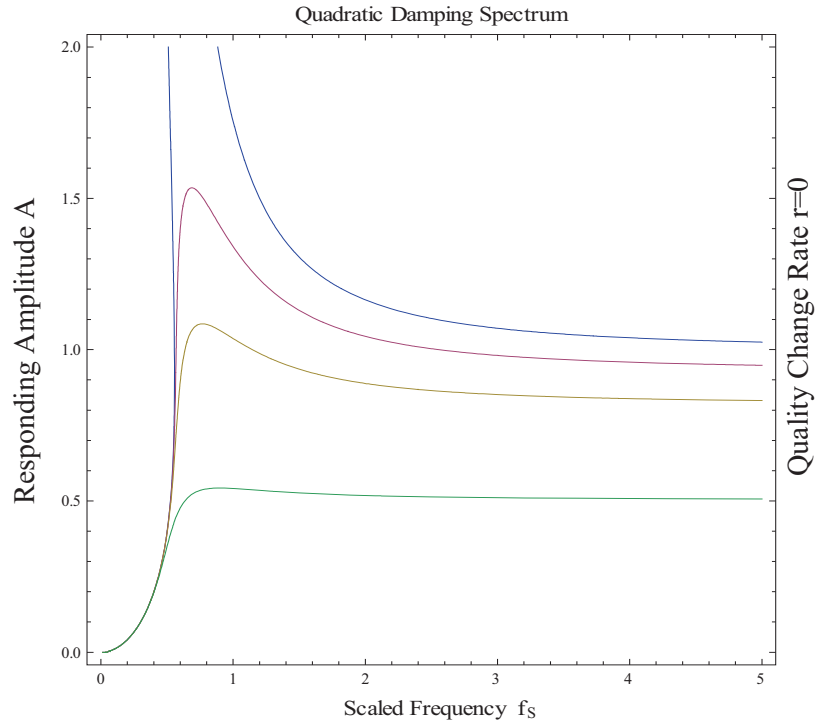

Figure 6: Forced oscillations with quadratic damping and constant quality value. 


\section{Experimental application example}

Eqn (27) defines the phase angle between the harmonic excitation and the responding oscillation. By solving the equation for the damping factor $\mathrm{D}$, eqn (27) transforms into eqn (29) with $\mathrm{D}$ as a function of $\varphi, A$, and $G$.

$$
D=\left|\frac{3 \pi G}{8 A^{2}} \sin (\varphi)\right| .
$$

The exciting amplitude $\mathrm{G}$, the responding amplitude $\mathrm{A}$ and the phase angle $\varphi$ can be measured during the isenthalpic oscillations. With these three values, the quadratic damping factor $\mathrm{D}$ can be calculated and experimentally determined. In general it is difficult to determine the damping factor in steady state oscillations. Using eqn (29) and the measured listed parameters the quadratic damping factor $\mathrm{D}$ can be found.

\section{Conclusion}

Pressure induced isenthalpic oscillations with condensation and evaporation in saturated two-phase fluids have stable solutions for the whole range of the saturated fluid within the saturation dome. The solution pattern is more complicated close to the vapour side of the saturation dome at negative slopes of the constant quality curve. There are no instabilities for free or forced oscillations. The effect of quadratic fluid damping reduces the responding amplitudes significantly.

\section{References}

[1] Yunus A. Cengel, Michael A. Boles, "Thermodynamics: An Engineering Approach," $3^{\text {rd }}$ Edition, McGraw-Hill, 1998.

[2] Tse, Francis S. et al. "Mechanical Vibrations", Allyn and Bacon Inc., Boston, MA 02210, 1978, ISBN 0-205-05940-6.

[3] Andrew Kimmel, "Pressure induced non-linear oscillations in two-phase LNG pipe flow," AIChE Spring National Meeting 2006, Orlando FL, April 23-27, 2006.

[4] Joel V. Madison et al. "Pressure Induced Isenthalpic Oscillations with Condensation and Evaporation in Saturated Two-Phase Fluids" ICFM 2010 International Conference on Fluid Mechanics, September 28-30, 2010, Amsterdam, NL, Proceedings ISSN 1307-6892.

[5] Wolfram Mathematica 7, http://www.wolfram.com. 